\title{
Respuesta del sistema antioxidante en varones sanos, frente a hiperglicemia aguda inducida
}

\author{
Healthy males' antioxidant system response in induced acute hyperglycemia
}

\author{
Raquel Oré ${ }^{1}$, Oscar Castillo ${ }^{2}$, Miguel Sandoval', Rubén Valdivieso ${ }^{1}$, Rosa Oriondo ${ }^{1}$, \\ Orison O Woolcott ${ }^{2}$, Jorge Durand ${ }^{1}$, Lida Tello ${ }^{2}$ \\ ${ }^{1}$ Centro de Bioquímica y Nutrición, Facultad de Medicina, Universidad Nacional Mayor de San Marcos. Lima, Perú. \\ ${ }^{2}$ Instituto Nacional de Biología Andina, Facultad de Medicina, Universidad Nacional Mayor de San Marcos. Lima, Perú.
}

\begin{abstract}
Resumen
Objetivo: determinar la respuesta del sistema antioxidante en varones sanos, frente a la hiperglicemia aguda inducida. Diseño: estudio prospectivo, descriptivo, longitudinal, experimental. Lugar: Instituto Nacional de Biología Andina, Facultad de Medicina, Universidad Nacional Mayor de San Marcos, Lima, Perú. Material biológico: Sangre y suero de sujetos aparentemente sanos. Intervenciones: A 13 sujetos adultos clínicamente sanos, entre 20 y 41 años, después de 10 horas de ayuno, se administró glucosa vía endovenosa, mediante el método de clamp hiperglicémico, a $125 \mathrm{mg} / \mathrm{dL}$ por encima del valor basal, durante 120 minutos. Se realizó mediciones de la glicemia a $0,5,10,15,20,30,40,50,60,70,80,90$, 100,110 y 120 minutos. Se tomó la muestra sanguínea con anticoagulante EDTA y otra de sangre total, para obtención de suero, para las pruebas bioquímicas a los 0,60 y 120 minutos. Principales medidas de resultados: Modificaciones de la glicemia y lipoperoxidación en suero, glutatión y actividad superóxido dismutasa en glóbulos rojos lisados e índices de estrés oxidativo. Resultados: $\mathrm{EI}$ nivel de glucosa durante el clamp hiperglicémico, luego de alcanzar el 'equilibrio', fue $197 \pm 17,58 \mathrm{mg} / \mathrm{dL}$. La lipoperoxidación aumentó de 2,54 + 0,51 a 2,90 + $0,58 \mathrm{umol} / \mathrm{L}$, de 0 a 60 minutos, y a 2,66 + 0,55 umol/L a los 120 minutos. El glutatión se redujo en $8,10 \%$ a la hora, aumentando $7,08 \%$ a los 120 minutos. La actividad superóxido dismutasa se elevó $0,54 \%$ a los 60 minutos y $5,66 \%$ a los 120 minutos, sobre el basal. Los índices de valoración del estrés oxidativo tuvieron correlación r Pearson positiva, en nivel alto a muy alto. Conclusiones: la hiperglicemia aguda inducida hasta 2 horas elevó el estrés oxidativo, promoviendo generación de defensa antioxidante, con síntesis de glutatión reducido de novo y mayor actividad de la superóxido dismutasa.
\end{abstract}

Palabras clave: Técnica de clampeo de la glucosa; estrés oxidativo; glutatión; peroxidación de lípido; superóxido dismutasa.

\begin{abstract}
Objective: To determine healthy males' antioxidant system response in induced acute hyperglycemia. Design: Prospective, descriptive, longitudinal, experimental study. Setting: National Institute of Andean Biology, Faculty of Medicine, Universidad Nacional Mayor de San Marcos, Lima, Peru. Biological materials: Whole blood and serum of apparently healthy men. Interventions: After 10 hour fasting, intravenous glucose was administered to thirteen healthy 20-41 year-old adult men using the hyperglycemic clamp method at $125 \mathrm{mg} / \mathrm{dL}$ above basal value during 120 minutes. Glycemia was measured at $0,5,10,15,20,30,40$, $50,60,70,80,90,100,110$, and 120 minutes. Blood sampling was obtained with EDTA anticoagulant and whole blood to obtain blood serum for biochemistry tests at 0,60 and 120 minutes. Main outcome measures: Blood serum glycemia and lipoperoxidation variation, glutathione and superoxide dismutase activity in lysed red blood cells and oxidative stress index. Results: After achieving 'balance', glucose levels during hyperglycemic clamp was $197 \pm 17,58 \mathrm{mg} / \mathrm{dL}$. Lipoperoxidation increased from $2,54+0,51$ to $2,90+0,58$ umol/L, from 0 to 60 minutes, and to $2,66+0,55 \mathrm{umol} / \mathrm{L}$ at 120 minutes. Glutathione was reduced in $8,10 \%$ at one hour, rising $7,08 \%$ at 120 minutes. Superoxide dismutase activity rose above basal $0,54 \%$ at $60 \mathrm{~min}$ and $5,66 \%$ at $120 \mathrm{~min}$. Oxidative stress valuation index had high level to very high level positive Pearson $r$ correlation. Conclusions: Acute induced hyperglycemia up to 2 hours increased oxidative stress, promoting generation of antioxidant defence with de novo synthesis of reduced glutathione and greater activity of superoxide dismutase.
\end{abstract}

Key words: Glucose clamp technique; oxidative stress; glutathione; lipid peroxidation; superoxide dismutase.

\section{INTRODUCCIÓN}

La hiperglicemia es el incremento de la glucosa sanguínea sobre los valores estimados como normales. Este incremento puede ser silente cuando los niveles séricos no son ligeramente elevados o manifestar sintomatología, cuando la elevación es muy por encima del valor normal. En ambos casos, la hiperglicemia podría producir alteración del equilibrio oxidativo, generando daño o estrés oxidativo, hipótesis que motivó el desarrollo del presente estudio. El daño o estrés oxidativo ha sido definido como la exposición del organismo a diversas fuentes que producen una rotura del equilibrio que debe existir entre las sustancias o factores prooxidantes y los mecanismos antioxidantes encargados de eliminar dichas especies químicas, ya sea por un déficit de estas defensas o por un incremento exagerado de la producción de especies reactivas derivadas del oxígeno (EROs). La producción excesiva de estas especies produce daño celular, trastornos fisiológicos y cambios patológicos ${ }^{(1)}$.

Las reacciones oxidativas son una fuente potencial citotóxica continua de EROs, las cuales tienen rol importante en los sistemas biológicos ${ }^{(1)}$.

Las principales EROs son el radical superóxido $\left(\mathrm{O}_{2}^{-}\right)^{-}$, el peróxido de hidró- geno $\left(\mathrm{H}_{2} \mathrm{O}_{2}\right)$ y el radical oxidrilo (HO-). Una de las principales fuentes de EROs es la cadena respiratoria, donde pueden ocurrir las siguientes transferencias de electrones:

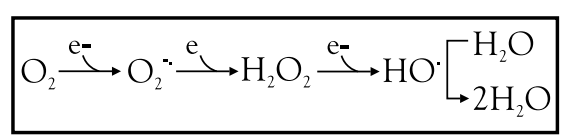

En la cadena respiratoria, aproximadamente 3\% de los electrones provenientes del nicotinamida adenina dinucleótido reducido $\left(\mathrm{NADH}+\mathrm{H}^{+}\right.$) se desvía hacia la formación de EROs, por la reducción incompleta del oxígeno. 
$\mathrm{El} \mathrm{O}_{2}^{--}$es una de las principales EROs en las células; la formación de este radical también puede ocurrir a nivel de la NADPH-oxidasa, según la siguiente reacción:

$$
2 \mathrm{O}_{2}+\mathrm{NADPH} \rightarrow 2 \mathrm{O}_{2}^{-\cdot}+\mathrm{NADP}^{+}+\mathrm{H}^{+}
$$

El $\left(\mathrm{O}_{2}^{-}\right)$se une a proteínas, formando un complejo, el que por estrés oxidativo sufre modificaciones de conformación, exponiendo distintos sitios de interacción proteica, que le permiten unirse a 2 ferroproteínas integrales de membrana. De esta manera, queda formado un complejo proteico con actividad NADPH-oxidasa (2). La activación de este complejo está mediada por el sistema Ras unido a la (guanosina trifosfato) GTP.

$\mathrm{El}_{2} \mathrm{O}_{2}$ puede reaccionar con metales divalentes (libres o unidos a proteínas) y producir HO, vía reacción de Fenton. El ejemplo tipo es la reacción con $\mathrm{Fe}^{++}$ libre, que ocurre según la siguiente reacción ${ }^{(2)}$ :

$$
\mathrm{Fe}^{++}+\mathrm{H}^{+}+\mathrm{H}_{2} \mathrm{O}_{2} \longrightarrow \begin{array}{l|}
\mathrm{Fe}^{++}+\mathrm{HO}+\mathrm{H}_{2} \mathrm{O} \\
\text { (principalmente en citosol) }
\end{array}
$$

En forma similar, puede reaccionar también con el grupo prostético de metaloproteínas que contienen hierro (ej. con la dihidroxiácido dehidrasa, la 6 fosfogluconato dehidrasa, las fumarasas A y B o la aconitasa), según la reacción de Haber Weis ${ }^{(2)}$ :

$$
\begin{array}{|l}
\frac{\mathrm{Fe}^{+++}-\text {complejo }+\mathrm{O}_{2}^{*} \longrightarrow \mathrm{Fe}^{++}-\text {complejo }+\mathrm{O}_{2}}{\mathrm{Fe}^{++} \text {- complejo }+\mathrm{H}_{2} \mathrm{O}_{2} \rightarrow \mathrm{HO}+\mathrm{HO}^{+}+\mathrm{Fe}^{+++} \text {- complejo }} \\
\mathrm{O}_{2}^{-}+\mathrm{H}_{2} \mathrm{O}_{2} \longrightarrow \mathrm{O}_{2}+\mathrm{HO}+\mathrm{HO}^{-}
\end{array}
$$

El HO puede reaccionar con distintas macromoléculas (proteínas, lípidos y ácidos nucleicos, principalmente), en las que por cesión de un electrón se producen otras especies reactivas, a través de mecanismos aún desconocidos. En estos casos, se dice que ha intervenido el radical oxidrilo, entendiendo como tal a un radical proveniente de oxidaciones univalentes, iniciadas por una reacción de tipo Fenton ${ }^{(3)}$. En este tipo de reacciones, la hidroxila- ción y la abstracción de hidrógeno son las modificaciones más comunes que sufre el sustrato orgánico involucrado y se generan otros radicales libres orgánicos, tales como los radicales alcohoxilos ( $\mathrm{RO} \cdot)$, peroxilos (ROO) y sulfoderivados ${ }^{(2)}$.

Existen otras dos EROs, con características especiales: el oxígeno singulete $\left({ }^{1} \mathrm{O}_{2}\right)$ y el hipoclorito (en su forma no protonada $\mathrm{ClO}^{-}$o protonada, llamado también ácido hidrocloroso). Otra fuente de EROs está relacionada a la dupla xantino/xantino oxidasa. La acumulación de hipoxantina y xantina, bajo condiciones anaeróbicas, de isquemia/reperfusión (deficiencia en la irrigación sanguínea -que empobrece la llegada de sangre y, por consiguiente, de oxígeno a un tejido- con posterior reflujo sanguíneo y consecuente afluencia de oxígeno) o de contenido energético bajo, puede desembocar en la producción de EROs ${ }^{(2)}$.

Bajo condiciones fisiológicas, los EROs son inactivados por un sistema de defensa localizado tanto intra como extracelularmente. Sin embargo, en ciertas condiciones se incrementa la generación de EROs y/o la disminución de las defensas antioxidantes, generándose el daño oxidativo (2).

Si bien todos los organismos vivos soportan numerosos factores endógenos y exógenos de estrés oxidativo, al mismo tiempo poseen numerosos sistemas de defensas antioxidantes regulables, enzimáticos y no enzimáticos. Existen enzimas que actúan específicamente sobre determinadas especies reactivas. Así, la superóxido dismutasa (SOD) dismuta al $\mathrm{O}_{2}^{-\cdot}$ a $\mathrm{O}_{2}$ y $\mathrm{H}_{2} \mathrm{O}_{2}$, la catalasa transforma al $\mathrm{H}_{2} \mathrm{O}_{2}$ en $\mathrm{O}_{2}$ y agua, la GSH-peroxidasa cataliza la reducción de peróxidos $(\mathrm{ROOH}$, inclusive al $\mathrm{H}_{2} \mathrm{O}_{2}$ ) a alcoholes $(\mathrm{ROH})$, aprovechando el potencial reductor del GSH. Existen otras enzimas, tales como las quinonas reductasas y hemooxigenasa, que pueden prevenir la formación de EROs, por ciclado de electrones ${ }^{(4)}$. Algunos metales, tales como el Se y el $\mathrm{Zn}$, por su participación como cofactores de enzimas antioxidantes (GPx y SOD citoplasmática, respectivamente), contribuyen a aumentar las defensas antioxidantes ${ }^{(2)}$.
Entre las defensas antioxidantes no enzimáticas, tiene un lugar predominante el glutatión (GSH). Este tripéptido (GSH, g-L-glutamil-L-cisteinil-glicina) protege a la célula contra diferentes especies oxidantes y se ha comprobado su participación clave en numerosos desórdenes neurodegenerativos. Tanto el GSH como otras moléculas conteniendo tioles tienen alto poder reductor y, por consiguiente, poseen propiedades antioxidantes, ya que pueden cederle un electrón a las EROs y/o ERNs, disminuyendo de esta forma su reactividad. Se dice que este tipo de compuestos de peso molecular bajo actúan como 'depuradores' de radicales libres. Entre ellos podemos citar a la tiorredoxina (Trx) y a la vitamina C o ácido ascórbico (hidrosoluble) y a las vitaminas liposolubles E y la vitamina $\mathrm{A}^{(2)}$.

Estudios epidemiológicos indican que la ingestión de frutas y vegetales confiere protección contra el desarrollo de cáncer y de otras enfermedades frecuentemente asociadas a estrés oxidativo. Se ha propuesto que el efecto benéfico de este tipo de alimentos radica en las propiedades antioxidantes de las vitaminas y a la presencia de compuestos como los polifenoles (con propiedades de capturar radicales libres y quelantes de metales) ${ }^{(2)}$.

Además de los mecanismos de protección antioxidante, enzimáticos y no enzimáticos, también contribuyen a paliar el posible daño oxidativo a través de tres mecanismos: a) el 3\% del oxígeno de la respiración se transforma en $\mathrm{O}_{2}^{-}$- mediante una reducción univalente, que ocurriría en el complejo I y en el complejo III mitocondrial ${ }^{(5,6)}$; b) la compartimentalización celular, lo que trae como consecuencia que las EROs y sus fuentes no siempre estén cerca de sus blancos de acción; y, c) varios factores estructurales de los ácidos nucleicos favorecen su protección ante el estrés oxidativo: la cromatina compacta, la presencia de histonas y la formación de complejos estables del ADN con las proteínas básicas y no básicas ${ }^{(2)}$.

Las investigaciones en marcha están tratando de explicar la participación de las EROs en el desarrollo y características clínicas de varias enfermedades, tales como diabetes, cirrosis alcohólica, hiper- 
tiroidismo, cáncer, infarto al miocardio, entre otras ${ }^{(7)}$.

Siendo la hiperglicemia crónica una característica común de la diabetes no controlada, esta condición puede conllevar al incremento de la producción de EROs, con una reducción de las defensas antioxidantes y alteración del estado redox intracelular del organismo, lo cual iniciaría una serie de eventos moleculares complejos responsables de las complicaciones diabéticas ${ }^{(8)}$. La hiperglicemia condiciona una sobreproducción de EROs, que afectaría a macromoléculas como los ácidos grasos insaturados de las membranas biológicas (medido por lipoperoxidación: mediante la formación del complejo especies reactivas derivadas del ácido tiobarbitúrico malondialdehido (MDA-TBARS), dienos conjugados, también a proteínas (glicación de proteínas) y especialmente el daño al ADN (8-oxoguanina).

El objetivo del presente trabajo fue determinar la respuesta del sistema antioxidante in vivo, frente a la hiperglicemia aguda inducida, en varones sanos, utilizando el método de clamp hiperglicémico, para observar la respuesta del organismo frente a la elevación de la glucosa en sangre ya que en pacientes con diabetes se observa una depleción de las defensas antioxidantes, desconociéndose lo que ocurre funcionalmente en condiciones normales.

\section{MÉTODOS}

Se estudió 13 varones, voluntarios, clínicamente sanos, residentes en Lima. El estudio se realizó en el Instituto Nacional de Biología Andina, Facultad de Medicina, Universidad Nacional Mayor de San Marcos, Lima, Perú en el periodo 2004-2005.

Previo ayuno mínimo de 10 horas, se realizó el clamp hiperglicémico, el cual consistió en elevar los niveles de glucosa sanguínea a $125 \mathrm{mg} / \mathrm{dL}$ por encima del valor basal, según el método de De Fronzo $(1979)^{(9)}$. Para ello, se colocó a los voluntarios un catéter en cada antebrazo; uno de ellos se utilizó para la infusión de dextrosa al $20 \%$ y el otro para la obtención de sangre para las mediciones de lipoperoxidación, glutatión reducido y superóxido dismutasa. La glicemia fue realizada tomando sangre capilar y medida con tiras reactivas.

Se usó un software GWBASIC.EXE, que nos permitió elevar la glicemia a la concentración deseada y luego mantenerla durante las dos horas que duró la prueba. El software GWBASIC.EXE es un programa matemático informático utilizado para corregir la velocidad de infusión de la dextrosa al 20\%. El valor de glicemia capilar obtenido a partir del minuto 20 y de allí cada 10 minutos hasta el minuto 110, fue introducido en la computadora, la cual a través del programa nos indica la velocidad de infusión a corregir para mantener el valor de la glicemia deseada, en los tiempos programados.

Se obtuvo muestras de sangre para llevar a cabo las siguientes determinaciones:

- Glicemia a los -15, 0, 5, 10, 15, 20, $30,40,50,60,70,80,90,100,110$ y 120 minutos, con el método de glucosa oxidasa, con tiras reactivas Boehringer Mannheim.

- Lipoperoxidación (LPO) en suero fresco, según el método de Buege ${ }^{(10)}$ modificado por Suárez ${ }^{(11)}$, a los 0, 60 y 120 minutos, midiendo en el espectrofotómetro la formación del complejo tiobarbitúrico-malondialdehido (MDA) a $535 \mathrm{~nm}$.

- Separación de eritrocitos y hemólisis, para la determinación de glutatión (GSH) y actividad de la enzima superoxidismutasa (SOD); se usó una muestra de sangre con EDTA como anticoagulante; luego se lavó los eritrocitos en suero fisiológico (solución de $\mathrm{NaCl} 0,9 \mathrm{~g} / \mathrm{dL}$ ) y se centrifugó por 8 minutos, a 3000 RPM; se eliminó el sobrenadante, se resuspendió con suero fisiológico y se centrifugó nuevamente; se repitió esta operación hasta obtener sobrenadantes claros; se eliminó el sobrenadante y se resuspendió los hematíes del precipitado con agua helada, que fueron colocados en refrigeración, para la hemolización.
- Se midió glutatión reducido (GSH) total, según el método de Boyne ${ }^{(12)}$, a los 0, 60 y 120 minutos; se determinó en eritrocitos hemolizados, en presencia de vitamina C, glioxilato y ácido 2 ditiobisnitrobenzoico (DTNB), midiendo en el espectrofotómetro a $412 \mathrm{~nm}$.

- La actividad superóxido dismutasa (SOD) se determinó según el método de Marklund ${ }^{(13)}$, a los 0, 60 y $120 \mathrm{mi}$ nutos, y fue medida en los eritrocitos hemolizados utilizando como control la autooxidación del pirogalol en buffer tris- $\mathrm{HCl}$, a $420 \mathrm{~nm}$.

A partir de los datos de glicemia e indicadores del estrés oxidativo -como lipoperoxidaciòn sérica, actividad superóxido dismutasa y concentración de glutatión reducido-, se obtuvo el promedio aritmético, la desviación estándar muestral y rango: valores máximo y mínimo. También, se calculó la variación porcentual de los indicadores del estrés oxidativo en sangre, tomando el cambio que hubo a los 60 y 120 minutos, así como cien por ciento el valor de 60 minutos, para comparar la variación a los 120 minutos. Se calculó además índices de la valoración del estrés oxidativo en sangre, como: glicemia/lipoperoxidación, actividad SOD/lipoperoxidación y concentración de glutatión/lipoperoxidación, en los tiempos basal, 60 y 120 minutos.

Los resultados fueron sometidos al análisis de correlación de la r de Pearson, teniendo la siguiente consideración: si la correlación era menor a 0,20, la correlación era muy pequeña, prácticamente despreciable; si el valor estaba entre 0,20 y 0,39, la correlación era baja; entre 0,40 y 0,59 , la correlación era moderada; con valores entre 0,60 y 0,80, la correlación era alta o marcada; y si la correlación era mayor que 0,80, entonces era muy alta. El análisis se realizó para una significancia mínima de 0,05, mediante el cálculo del error típico y la razón típica, para lo cual se usó el programa SPSS, versión 15,0 .

Se contó con el consentimiento informado de los pacientes, requisito necesario en el periodo que se realizó este estudio. 


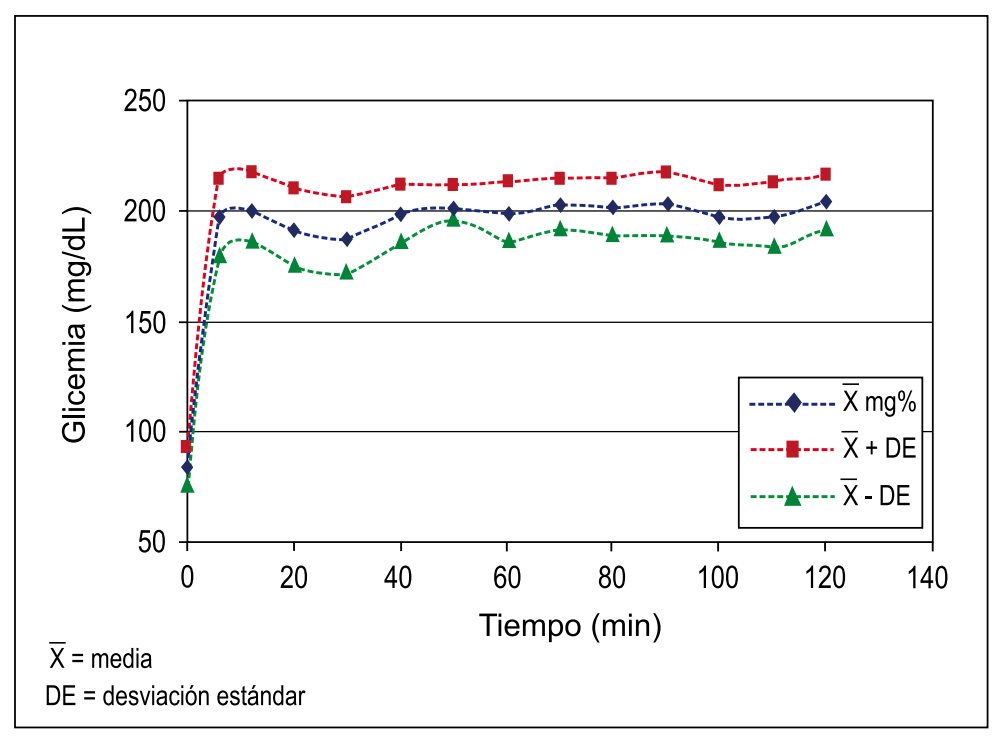

Figura 1. Niveles inducidos de glucosa sanguínea.

\section{RESULTADOS}

Los sujetos de investigación presentaron edades entre 20 y 41 años, promedio $28,2 \pm 6,5$, índice de masa corporal (IMC) entre 22,6 y $26,5 \mathrm{~kg} / \mathrm{m}^{2}$, promedio $23,8 \pm 1,2$. Los niveles de glucosa sanguínea basal en ayunas fueron $84,4 \pm 8,4 \mathrm{mg} /$ dL. A los 6 minutos de la administración de glucosa endovenosa, la elevación de la glicemia fue en promedio $197,4 \pm 17,58$ $\mathrm{mg} / \mathrm{dL}$ y se mantuvo en valores de 200 $\mathrm{mg} / \mathrm{dL}$ hasta los 120 minutos, como se observa en la figura 1. No se encontró relación entre los niveles de glicemia basal con el IMC.

De la misma manera, los valores de glicemia basal a los 6 minutos, así como la cantidad de glucosa administrada, no tuvieron correlación con otros indicadores. No se informó sobre algún efecto adverso en los sujetos voluntarios, durante ni después de la hiperglicemia inducida.

En la figura 1, se observa la curva de los niveles de glucosa en sangre durante la administración de glucosa endovenosa para inducir hiperglicemia, durante 120 minutos. En la medición basal (tiempo $0)$, todos los sujetos de estudio presentaron niveles en el rango considerado normal.

El nivel de estrés oxidativo en sangre, observado por lipoperoxidación, aumentó de 2,54 $\pm 0,51$ a $2,90 \pm 0,58 \mathrm{umol} / \mathrm{L}$, de 0 a 60 minutos, para descender a 2,66 $\pm 0,55$ a los 120 minutos de la prueba (tabla 1). La defensa antioxidante generada en el organismo de los voluntarios fue evaluada por la SOD, la cual mostró elevación del valor basal a los 60 minutos, para luego reducirse a los 120 minutos. La concentración de glutatión se redujo a los 60 minutos, en comparación al valor basal, y luego se elevó, sin superar el nivel basal.

Los cambios tanto del estrés oxidativo, medido por lipoperoxidación, como la defensa antioxidante, determinada por actividad SOD y glutatión, fueron valorados de manera adimensional por estimación de la variación porcentual,
$\mathrm{DE}=$ desviación estándar. mostrándose variación significativa a los 120 minutos, comparada con el valor basal o el valor de 60 minutos (tabla 2); para los valores de lipoperoxidación esta variación determinó un incremento promedio de $15,2 \%$ a los 60 minutos respecto al basal y se redujo a $-7,3 \%$ a los 120 minutos; y, para los valores de la SOD, se observó un incremento de $5,7 \%$ a los 120 minutos respecto al valor basal y de 4,5\% respecto al valor de 60 minutos; para ambos casos la correlación fue alta.

Respecto al glutatión, a los 60 minutos se observó disminución de su concentración en $8,1 \%$ y elevación a los $120 \mathrm{mi}$ nutos de $7,1 \%$; estas variaciones tuvieron correlación significativa (tabla 2).

La valoración del la lipoperoxidación en función de la glicemia basal no tuvo correlación. Las relaciones de la actividad superóxido dismutasa con lipoperoxidación y glutatión con lipoperoxidación fueron de un nivel marcadamente alto a muy alto.

Para el caso de la relación SOD/lipoperoxidación, hubo un descenso sostenido desde el tiempo cero o basal, hasta los 120 minutos. Mientras que, para la relación glutatión/ lipoperoxidación, se observó una reducción a los 60 minutos, para subir a los 120 minutos, sin llegar a la relación basal (ver tabla 3 ).

\section{DISCUSIÓN}

La prevalencia de diabetes mellitus se incrementa en forma muy rápida y se considera que, para el año 2025, la cifra

Tabla 1. Niveles de estrés oxidativo en sangre.

\begin{tabular}{lcccc}
\hline Indicadores de estrés oxidativo & Promedio & DE & Valor máximo & Valor mínimo \\
\hline Lipoperoxidación basal $\mu \mathrm{mol} / \mathrm{L}$ & 2,54 & 0,51 & 3,42 & 1,81 \\
Lipoperoxidación $60 \mathrm{~min} \mu \mathrm{mol} / \mathrm{L}$ & 2,90 & 0,58 & 3,77 & 2,27 \\
Lipoperoxidación $120 \mathrm{~min} \mu \mathrm{mol} / \mathrm{L}$ & 2,66 & 0,55 & 3,85 & 2,04 \\
SOD basal UI/mL & 859,7 & 424,5 & 1270,6 & 329,4 \\
$\mathrm{SOD} 60 \mathrm{~min} \mathrm{UI/mL}$ & 865,8 & 459,3 & 1651,7 & 411,76 \\
SOD $120 \mathrm{~min} \mathrm{UI/mL}$ & 742,5 & 426,8 & 1087, & 494,12 \\
Glutatión basal mg/dL & 665,6 & 117,7 & 845,9 & 533,5 \\
Glutatión $60 \mathrm{~min} \mathrm{mg} / \mathrm{dL}$ & 626,1 & 148,9 & 871,9 & 383,9 \\
Glutatión $120 \mathrm{~min} \mathrm{mg} / \mathrm{dL}$ & 643,1 & 123,7 & 819,8 & 481,1 \\
\hline
\end{tabular}


Tabla 2. Variación porcentual de los indicadores del estrés oxidativo en sangre.

\begin{tabular}{|c|c|c|c|c|c|}
\hline Variación \% del estrés oxidativo & Promedio & $\mathrm{DE}$ & Valor máximo & Valor mínimo & r Pearson \\
\hline Lipoperoxidación 60/basal * & 15,2 & 17,7 & 54,2 & $-9,6$ & 0,77 \\
\hline 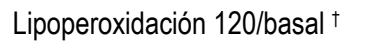 & 4,8 & 9,3 & 16,3 & $-17,3$ & 0,88 \\
\hline Lipoperoxidación 120/60 * & $-7,3$ & 15,0 & 16,5 & $-32,9$ & 0,64 \\
\hline SOD 60/basal ${ }^{\dagger}$ & 0,5 & 19,5 & 29,9 & $-18,2$ & 0,92 \\
\hline SOD 120/basal ${ }^{\dagger}$ & 5,7 & 27,0 & 50,0 & $-29,2$ & 0,89 \\
\hline SOD $120 / 60$ * & 4,8 & 30,5 & 51,0 & $-34,2$ & 0,75 \\
\hline Glutatión $60 /$ basal $^{\dagger}$ & $-8,1$ & 10,4 & 11,5 & $-28,0$ & 0,94 \\
\hline 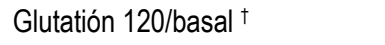 & $-2,7$ & 6,8 & 3,8 & $-10,0$ & 0,96 \\
\hline Glutatión 120/60 † & 7,1 & 13,4 & 25,4 & $-7,2$ & 0,92 \\
\hline
\end{tabular}

$\mathrm{DE}=$ desviación estándar.

* Análisis de correlación r, positivo, nivel alto o marcado.

† Análisis de correlación r, positivo, nivel moderado.

\begin{tabular}{|c|c|c|c|c|c|}
\hline Îndices valoración del estrés oxidativo & Promedio & $\mathrm{DE}$ & Valor máximo & Valor mínimo & r Pearson \\
\hline SOD 60/ lipoperoxidación 60 * & 283,8 & 140,1 & 500,2 & 164,7 & 0,56 \\
\hline SOD 120/ lipoperoxidación 120 * & 276,0 & 150,7 & 640,2 & 180,6 & 0,40 \\
\hline Glutatión basal/ lipoperoxidación basal ${ }^{\dagger}$ & 266,9 & 41,1 & 327,9 & 179,6 & 0,61 \\
\hline Glutatión 60/ lipoperoxidación $60^{\dagger}$ & 224,6 & 62,8 & 322,8 & 131,2 & 0,77 \\
\hline Glutatión $120 /$ lipoperoxidación 120 ₹ & 249,5 & 44,5 & 320,9 & 165,5 & 0,81 \\
\hline
\end{tabular}

de diabéticos a nivel mundial será de 333 millones, o sea $6,3 \%$ de la población. Si tenemos en cuenta que la mortalidad se eleva en este grupo de pacientes hasta 4 veces más que en sujetos no diabéticos, debido fundamentalmente a las manifestaciones tardías de la enfermedad, generadas por el daño macro y microvascular, es sumamente importante tratar de prevenirlas. Estudios clínicos con un número significativo de casos han demostrado que los pacientes que logran un mejor control metabólico presentan un daño vascular menor que aquellos con un control inadecuado, tanto en diabéticos de tipo 1 como de tipo 2 (UKPDS, DCCT) ${ }^{(14,15)}$. Actualmente, se acepta que este daño vascular tiene una relación íntima con la hiperglicemia crónica ${ }^{(1)}$.

Es conocido que la hiperglicemia crónica induce la generación de EROs,

Tabla 3. Índices de valoración del estrés oxidativo en sangre.

$\mathrm{DE}=$ desviación estándar.

* Análisis de correlación r, positivo, nivel moderado.

${ }^{\dagger}$ Análisis de correlación r, positivo, nivel alto o marcado.

‡ Análisis de correlación $r$ positivo, nivel muy alto.

las que producirían daño oxidativo, ocasionando incremento en la generación de $\mathrm{O}_{2} \cdot$ y de oxido nítrico $(\mathrm{NO})$. Este incremento es dañino, porque estas dos moléculas reaccionan y producen peroxinitrito, un potente oxidante que perdura mayor tiempo que los demás radicales libres ${ }^{(16,17)}$.

Estudios realizados por Marfella y col. ${ }^{(18)}$, así como Ceriello y col. ${ }^{(19)}$, encontraron que una hiperglicemia aguda inducida durante 120 minutos, mediante clamp hiperglicémico $(15 \mathrm{mmol} / \mathrm{L})$, causó estrés oxidativo, provocando una depleción de la capacidad antioxidante.

Por otro lado, existe la teoría que el estrés oxidativo está asociado con la autooxidación de la glucosa. Además, se postula cuatro mecanismos moleculares de daño tisular que produce la hiperglicemia; estos son: activación de la proteinquinasa $(\mathrm{PKC})$, que produciría la síntesis de novo de un segundo mensajero diacilglicerol (DAG), incremento del flujo de la vía de hexosaminas, incremento de productos de glicosilación avanzada (PGA) y elevación del flujo de la vía de los polioles ${ }^{(17,20)}$.

En nuestra investigación, se sometió a hiperglicemia aguda inducida a sujetos normales, durante 120 minutos; se les determinó un marcador de estrés oxidativo, midiendo la formación del complejo MDA - TBARS. Este parámetro fue valorado a nivel basal, 60 minutos y 120 minutos. La correlación de los 60 y 120 minutos con el nivel basal mostró una correlación alta (tabla 2). Frente a una agresión (estrés oxidativo) producida por la hiperglicemia aguda en sujetos normales, se observó en los primeros 60 minutos un incremento del marcador (peroxidación lipídica); sin embargo, al finalizar la prueba, este parámetro disminuyó; probablemente el organismo, frente a un daño celular, indujo la síntesis proteica de la enzima SOD y mejoró sus defensas antioxidantes. Estas variaciones han sido también observadas in vitro por Vincent y col. ${ }^{(21)}$.

Zorrilla-García y Fernández-Argones (22) realizaron un metaanálisis sobre diabetes y estrés oxidativo; mencionaron que en la diabetes mellitus descompensada aparecen y aumentan en el plasma sustancias como el malondialdehido, los dienos conjugados y las sustancias reactivas del ácido tiobarbitúrico, que son una medida de la peroxidación lipídica que se presenta en esta alteración metabólica, lo que evidencia la relación entre la diabetes mellitus y el estrés oxidativo. En nuestro trabajo, se observa la misma tendencia hacia la generación de estrés oxidativo, siendo la diferencia importante que los sujetos estudiados por nosotros eran sujetos normales sometidos a hiperglicemia aguda de 2 horas, no pacientes diabéticos, quienes dependiendo de su control metabólico generalmente están expuestos a hiperglicemia crónica.

En la revisión realizada por Dariel Díaz Arce ${ }^{(23)}$ sobre hiperglicemia y estrés oxidativo en cultivo de células, se menciona que un estudio realizado en 
células endoteliales de la vena umbilical expuestas a elevadas concentraciones de glucosa durante dos semanas mostraron una inducción en la expresión de las enzimas antioxidantes SOD, catalasa y glutatión peroxidasa, a partir del sétimo día de tratamiento. A diferencia de nuestro estudio, la hiperglicemia tuvo una duración de solamente 2 horas, observándose una misma tendencia en la respuesta antioxidante.

Cuando ocurre un desbalance entre la producción de radicales libres y las defensas antioxidantes, se produce el daño oxidativo. Frente a este tipo de agresión celular, el organismo sintetiza el GSH, tratando así de mantener el equilibrio redox y disminuir la cantidad de radicales libres ${ }^{(24)}$. Todo esto sugiere que la exposición a elevadas concentraciones de glucosa sanguínea puede generar un incremento de EROs, que reaccionan con los sistemas antioxidantes primarios del organismo, por ejemplo, el alfa tocoferol y el GSH, provocando disminución de las concentraciones de las defensas ${ }^{(23)}$.

La acción del glutatión reducido, como se ha mencionado, es el antioxidante más importante que tiene el organismo, porque permite amortiguar la agresión de EROs. En nuestro estudio, los niveles de glutatión reducido disminuyeron a los 60 minutos y luego se elevaron a los 120 minutos, sin llegar a los niveles basales (tabla 3); pero, si se toma en cuenta las variaciones porcentuales de cada hora en comparación con los niveles basales, se aprecia que realmente hay un descenso del nivel del GSH; esto nos permite deducir que el organismo, al defenderse frente a un daño oxidativo, consume rápidamente el antioxidante, por lo que inicialmente los niveles se reducen; sin embargo, el organismo sintetizaría GSH de novo, para lograr una mejor defensa antioxidante, lo que se observa a los 120 minutos, para luego elevar sus niveles a los 120 minutos.

En relación a la superóxido dismutasa, esta es la primera enzima antioxidante que cataliza la dismutación del radical citotóxico superóxido $\left(\mathrm{O}_{2}^{-}\right)$, formando oxígeno y peróxido de hidrógeno. Es la principal defensa antioxidante que presenta todo tipo de células que están expuestas al oxígeno. En nuestro estudio, esta enzima, frente a la elevación de la glicemia, mostró un incremento de su actividad para luego descender a valores promedios menores que el nivel basal; sin embargo, la variación porcentual fue de $5,7 \%$, habiendo una notoria elevación a los 120 minutos en comparación al basal. Estos resultados corroboran los estudios que indican que el organismo inicialmente usa sus defensas y, después de la persistente hiperglicemia, hay un incremento sostenido de la SOD.

Vincent y col. (21), en un estudio realizado en cultivos de neuronas de diabéticos, encontraron un incremento de la SOD y catalasa luego de 3 horas de incubación con una concentración de $20 \mathrm{mM}$ de glucosa. El incremento de la actividad de catalasa fue cinco veces respecto a los controles, mientras que el incremento de la SOD fue ligeramente moderado; así mismo, la concentración de GSH disminuyó significativamente. Esto sugiere que mientras el GSH está siendo oxidado a través del daño oxidativo, las neuronas son capaces de responder restituyendo este antioxidante; esto podría constituir una terapia contra la neuropatía diabética.

En nuestra investigación, el comportamiento de la variación de la SOD y GSH es semejante al estudio de Vincent (21), por lo que podríamos sugerir que el organismo, frente a una agresión como la hiperglicemia, inicialmente consume el GSH; luego, se regenera este antioxidante por síntesis de novo y también se incrementa la actividad de la SOD.

Por lo tanto, concluimos que la respuesta del organismo en varones sanos frente a la hiperglicemia aguda inducida hasta 2 horas eleva el estrés oxidativo, promoviendo generación de defensa antioxidante, con síntesis de glutatión reducido de novo y mayor actividad de la superóxido dismutasa.

\section{AGRADECIMIENTOS}

A la Fundación Alexander von Humboldt, Bonn-Alemania, por su apoyo en equipos para la técnica del clamp hiperglicémico.

\section{REFERENCIAS BIBLIOGRÁFICAS}

1. Goycheva P, Gapjeva P, Popov, B. Oxidative stress and its complications in diabetes mellitus. Trakia J Sci. 2006;4(1):1-8.

2. García Triana G. NADPH oxidasa fagocítica: características, ensamblaje y mecanismo de acción. Cap 2.2. Estrés oxidativo en Biomedicina. Libro electrónico. La Habana, Cuba: Ed. BiomedCECAM; 2001.

3. Fridovich I. Superoxide anion radical $\left(\mathrm{O}_{2}{ }^{-}\right)$. Superoxide dismutases and related matters. J Biol Chem. 1997;272:18515-7.

4. Rios de Molina M. El estrés oxidativo y el destino celular. Rev Química Viva. 2003;2:1.

5. Boveris A, Cadenas E, Stoppani OM. Role of ubiquinona in the mitochondrial generation of hydrogen peroxide. Biochem J. 1976;153:43544.

6. Turrens JF, Boveris A. Generation of superoxide anion by the NADH dehydrogenase of bovine heart mitochondria. Biochem J. 1980;1191:421-7.

7. Yang Z, Laubach V, French B, Kron I. Acute hyperglycemia enhances oxidative stress and exacerbates myocardial infarction by activating nicotinamide adenine dinucleotide phosphate oxidase during reperfusion. J Thorac Cardiovasc Surg. 2009;137(3):723-8.

8. Ceradini D, Yao D, Grogan R, Callaghan M, Edelstein D, Brownlee M, Gurtner G. Decreasing intracellular superoxide corrects defective ischemia-induced new vessel formation in diabetic mice. J Biol Chem. 2008;16:10930-8.

9. De Fronzo R, Tobin J, Andres R. Glucose clamp technique: a method for quantifying insulin secretion and resistance. Am J Physiol. 1979;237(3):E214-23.

10. Buege J, Aust S. Microsomal lipid peroxidation. Methods Enzymol. 1978;53:302-10.

11.Suárez S. Detoxificación hepática y defensa antioxidante por efecto de xenobióticos alimentarios. Tesis de Maestría en Bioquímica. Facultad de Medicina, UNMSM. Lima, Perú. 1995.

12. Boyne A, Ellman G. Assay for glutathione. Anal Biochem. 1972;46:639-53.

13. Marklund S, Marklund G. Involvement of the superoxide anion radical in the autoxidation of pyrogallol and a convenient assay for superoxide dismutase. Biochem. 1974;47:469-74.

14. Eastman R, Kahn R, Klein R, Lachin J, Lebovitz J, Harold ND, Vinicor F. Implications of the United Kingdom Prospective Diabetes Study. American Diabetes Association Position Statement. Diabetes Care. 2002;25(Supl 1):528-32.

15. Trial Reseach Group. The effect of intensive treatment of diabetes on the development and progression of long-term complications in insulin-dependent diabetes mellitus. N Engl J Med. 1993;329(14):977-86.

16. Ceriello A, Quagliaro L, Catone B, Pascon R, Piazzola M, Bais B, et al. Role of hyperglicemia in 
nitrotyrosine postprandial generation. Diabetes Care. 2002;25(8):1439-43.

17. Rolo A, Palmeira C. Diabetes and mitochondrial function: role of hyperglycemia and oxidative stress. Toxicol Appl Pharmacol. 2006;212(2):167-78.

18. Marfella R, Quagliaro L, Nappo F, Ceriello A, Giugliano D. Acute hyperglycemia induced and oxidative stress in healthy subjects. Letter to the Editor. J Clin Invest. 2001;108(4):635-6.

19. Ceriello A. Acute hyperglycemia and oxidative stress generation. Diabet Med. 1997;14:S45-9.

20. Aronson D. Hyperglycemia and the pathobiology of diabetic complications. Adv Cardiol. 2008;45:116.
21. Vincent AM, McLean LL, Backus C, Feldman EL. Short-term hyperglycemia produces oxidative damage and apoptosis in neurons. The FASEB Journal. FJ Express. 2005;19:638-40.

22.Zorrilla-García, Fernández-Argones. Diabetes mellitus y estrés oxidativo. Bioquimia. 1999;24(3):96.

23. Díaz D. Hiperglicemia y estrés oxidativo en el paciente diabético. Rev Cubana Invest Biomed. 2006;25(3):307-17.

24. Busquets $\mathrm{N}$. Efecto de una hepatectomia parcial sobre el estrés oxidativo en hígado de ratas. Estudios preliminares. Santa Fé: Universidad Nacional del Litoral; 2003.
Manuscrito recibido el 3 de agosto de 2009 y aceptado para publicación el 4 de setiembre de 2009.

Correspondencia:

Dra. Raquel Oré Sifuentes

Centro de Investigación en Bioquímica y Nutrición

Facultad de Medicina, UNMSM

Av. Grau 750. Lima 1, Perú

Correo-e: kelaore@yahoo.es 